\title{
Innovación docente con jóvenes interactivos: proyectos sociales reales y estrategias digitales para enseñar comunicación
}

\author{
Tamara BUENo DORAL \\ Universidad Complutense de Madrid \\ trbueno@pdi.ucm.es \\ Liisa Irene HÄNNINEN \\ Universidad Complutense de Madrid \\ liisaire@pdi.ucm.es
}

Recibido: 31/07/2012

Aceptado: 30/10/2012

\begin{abstract}
Resumen
La presente comunicación muestra los resultados obtenidos tras ocho años de aplicación y estudio de un método de innovación docente en la Facultad de Ciencias de la Información de la Universidad Complutense, adaptada a las necesidades de la juventud interactiva. Se trata de un método de enseñanza basado en proyectos sociales y profesionales reales, que contempla la interactividad desde una doble perspectiva: por un lado, considerando la universidad en la red como interacción online, capacidad que poseen los profesores y los estudiantes para aprovechar al máximo las múltiples oportunidades que brinda la comunicación digital al proceso educativo, y por otro, como universidad red, es decir, como posibilidad de interacción social entre el colectivo de docentes, estudiantes y el de las empresas e instituciones que conforman el mercado laboral.
\end{abstract}

Palabras clave: Red, comunicación interactiva, innovación docente, estrategia digital, proyecto social

\section{Educational innovation with interactive youth: teaching communication through real social projects and digital strategies}

\begin{abstract}
The present paper shows the results of a new educational innovation methodology, obtained after eight years of application and study at the Faculty of Communication Science, Complutense University of Madrid, and adapted to the requirements of interactive youth. The method is based on real social and professional projects and contemplates interactivity from a double point of view. On one hand, the university in the Web is seen as online interaction, the capacity of lecturers and students to obtain the maximum benefits from the multiple opportunities offered by digital communication to the educational process, and on the other, the university network as the ability of social interaction between the collective of professors, students and the companies and organizations forming the labor market.

Key words: Network, interactive communication, educational innovation, digital strategy, social project

\section{Referencia normalizada}

BUENO DORAL, Tamara y HÄNNINEN, Liisa Irene (2012): "Innovación docente con jóvenes interactivos: proyectos sociales reales y estrategias digitales para enseñar comunicación". Estudios sobre el mensaje periodístico. Vol. 18, núm. especial octubre, págs.: 161-170. Madrid, Servicio de Publicaciones de la Universidad Complutense.
\end{abstract}

Sumario: 1. Introducción: fuentes y estado de la cuestión. 2. Metodología para la innovación docente.3. Desarrollo: descripción de los estudios sobre el método y aplicaciones. 4. Conclusiones. 5. Referencias bibliográficas. 


\section{Introducción: fuentes y estado de la cuestión}

La universidad es permeable a los cambios tecnológicos, sociales, económicos y culturales que se producen en el mundo globalizado y en la sociedad del conocimiento, lejos de vivir al margen «del mundo real» como en ocasiones se le acusa. Sin embargo, las facultades de comunicación suelen ir a un ritmo más lento que las empresas y el mercado profesional en su adaptación a la las nuevas tecnologías de información y comunicación, debido a su coyuntura financiera y organizacional menos flexible. Todo ello a pesar de disponer del capital humano necesario para el cambio: tanto profesores como estudiantes dispuestos a sumergirse en el mundo de la innovación tecnológica y comunicacional, para trabajar con la realidad aumentada y simulada, aprovechar las oportunidades de la Web 2.0 y 3.0 y reciclarse constantemente para mantenerse al día en cuanto a las nuevas tecnologías. Actualmente la industria de la comunicación, más adaptada a la realidad del mercado sigue siendo el buque insignia en la red. Las agencias de publicidad y relaciones públicas, las centrales de medios, las agencias informativas y los medios de comunicación son los primeros en digerir la innovación en las TIC, tanto desde el punto de vista de soporte y canal de información, como desde el punto de vista de su aplicación estratégica en publicidad y periodismo. No obstante, en la enseñanza universitaria se está utilizando sólo parte del potencial interactivo de la Web 2.0 y aún menos de la Web 3.0.

La universidad en la red supone un espacio de acción real e interacción virtual, que supera los límites espaciales y temporales, logrando procesos de enseñanza y aprendizaje dinámicos que transcienden las dificultades de la docencia tradicional. Como resultado, los alumnos administran su propio proceso de aprendizaje, independiente o cooperativo, en el marco de un proyecto educativo determinado (García Aretio, 2001: 122). No obstante, la nueva sociedad de la información y el conocimiento se enfrenta a múltiples desafíos, y aunque no es el objeto de esta comunicación profundizar en esta cuestión, destacamos que algunas de las limitaciones más señaladas tanto por la literatura científica como por los organismos internacionales se refieren, a la formación de nuevos profesionales con el fin de asegurar su inserción en los mercados laborales crecientes, así como a la importancia de promover sistemas educativos flexibles que favorezcan el aprendizaje cooperativo y personalizado (UNESCO, 1995 y 1998).

Asimismo la universidad entendida como red social forma un marco idóneo para la innovación en la enseñanza de comunicación debido a que se trata de un campo profesional cuya razón de ser fundamental son las redes: la interrelación entre entidades, agencias de comunicación, medios masivos y públicos. Actualmente, la relevancia social de cualquier unidad social está condicionada por su presencia en las redes específicas (Castells, 2009: 19-23), tanto tecnológicas como sociales, y la universidad no es una excepción. En el gráfico adjunto se puede observar la doble interacción de la universidad, desde la perspectiva de ciencias de la información y especialmente desde la publicidad y las relaciones públicas. Por un lado, la facultad se benéfica de las capacidades instrumentales de la red: las plataformas de trabajo compartidas, los sistemas de almacenamiento y gestión de datos y las herramientas para la divulgación de proyectos. Así mismo, los alumnos diseñan campañas de co- 
municación que utilizan la red como soporte publicitario y canal de comunicación, especialmente las redes sociales, pero también las herramientas más consolidadas como pueden ser las Webs corporativas, «los microsites» de campañas y los blogs.

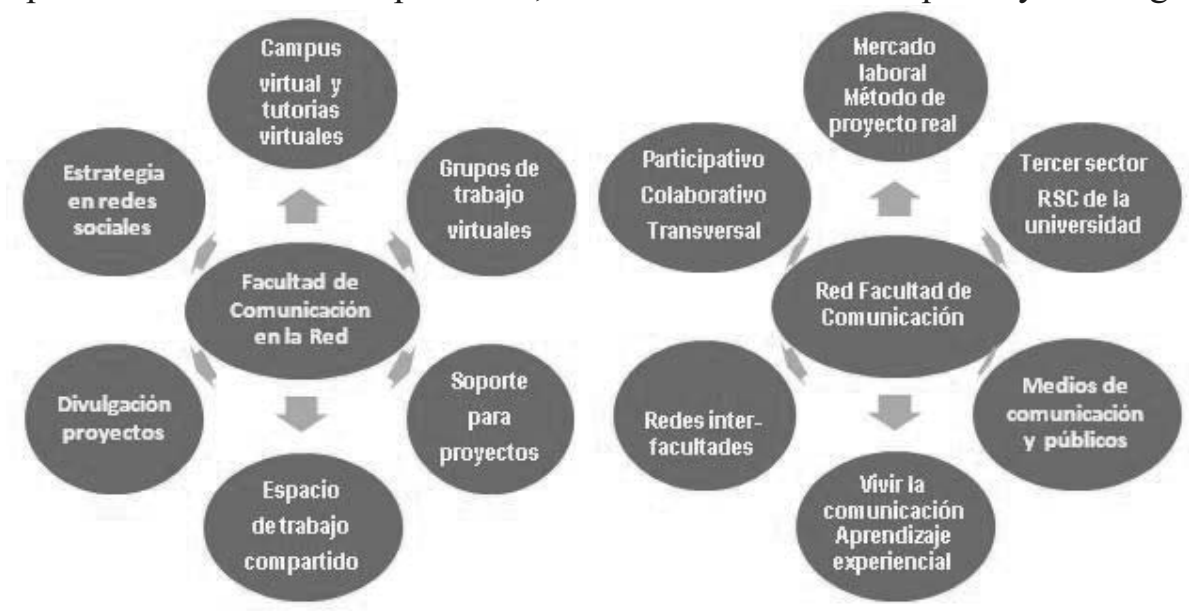

Figura 1: La interacción en su significado doble. Fuente: elaboración propia.

Por un lado, cuando se utilizan métodos docentes participativos y colaborativos que construyen una relación transversal con los agentes sociales externos, la facultad no sólo observa y analiza, sino que vive en directo la comunicación. De esta manera, la facultad interactúa con el mercado laboral, el tercer sector, los medios masivos y el público objetivo de las campañas. Cuando se combinan ambos tipos de interacción, se generan sinergias que facilitan el proceso de enseñanza y convierten a los estudiantes en los protagonistas de su propio aprendizaje. La base está en una correcta interacción social entre docentes y alumnos, una visión de profesores como supervisores que faciliten un clima de trabajo armonioso, aprendiendo a escuchar a los estudiantes y siendo proactivos en cuanto a las inquietudes de los jóvenes.

\section{Metodología para la innovación docente}

A raíz de más de ocho años de experiencias docentes en la aplicación de métodos interactivos, el grupo ha podido comprobar que la estrategia «Learning by doing» (aprender haciendo) por sí sola no permite a los alumnos alcanzar niveles de excelencia si los alumnos no están motivados y emocionalmente implicados con el proyecto docente, además de disponer de un marco de conocimientos teóricos y técnicos previos que les capaciten para realizar trabajos profesionales. Con el fin de mejorar la educación de los alumnos, las autoras han combinado la metodología del aprendizaje experiencial con el «método de proyecto social real» con, así como algunas técnicas del modelo de aprendizaje colaborativo, obteniendo resultados muy positivos año tras año. Así mismo, ambos enfoques están alineados con las exigencias del Espacio Europeo de Educación Superior, al tratarse de formulas donde la interacción entre estudiantes y profesores supone el principal motor para generar aprendizaje (Consejo de Coordinación Universitaria, 2006:7). 
La primera metodología empleada se basa en la «Teoría del aprendizaje experiencial» (Kolb, 1984:141), puesto que supone una base teórica muy consolidada, respaldada por innumerables estudios empíricos realizados a nivel internacional durante las dos últimas décadas. Dentro de este amplio marco, el «Project based learning» (PBL, Proceso de aprendizaje basado en proyectos), constituye el método seleccionado para el proyecto docente que se presenta en la comunicación. Entre otras ventajas, el PBL promueve un aprendizaje activo, crítico y creativo, mediante el cuál los alumnos se comprometen con su proceso de aprendizaje. Además, favorece que se desarrollen otras competencias trasversales y supone un elemento fundamental para la motivación de los alumnos. Con relación a la universidad en la red, se comprueba que esta propuesta docente favorece que los alumnos experimenten con las diversas posibilidades que les ofrecen los entornos virtuales aplicados al ámbito educativo. Por otro lado, durante la fase del diseño metodológico del proyecto se ha incluido el modelo de aprendizaje colaborativo, entendiendo que en el ámbito publicitario resulta esencial el trabajo en equipo, y además, el trabajo cooperativo es uno de los desafíos de la sociedad de la información y el conocimiento. El aprendizaje colaborativo es un sistema de interacciones cuidadosamente diseñado que organiza e induce la influencia recíproca entre los integrantes de un equipo. Es también un proceso en el que se va desarrollando gradualmente, entre los integrantes de dicho equipo, el concepto de ser «mutuamente responsables del aprendizaje de cada uno de los demás» (Johnson y Johnson, 1999:1). En la práctica las permanentes actividades planteadas en grupo, implican que los alumnos trabajen apoyándose unos a otros, aprendiendo a escucharse y a valorar las propuestas de los demás de una manera crítica y constructiva, con el fin de obtener un aprendizaje conjunto más completo que complemente al trabajo individual.

En cuanto al «Método docente de proyecto social real», éste se aplica de forma sistemática desde el curso 2003/4 en la Facultad de Ciencias de la Información (Núñez y Hänninen, 2010:4416), aunque el aprendizaje basado en proyectos sociales y comunicación se ha desarrollado paralelamente en otras universidades, como por ejemplo en la Pontificia Universidad Católica de Perú. Así mismo, las aportaciones de Saura desde la Universidad Pontificia Comillas fueron interesantes para desarrollar el aspecto solidario y educativo del método docente (Saura, 2007). En vez de aprender mediante simulacros, se pretende hacer a los alumnos partícipes de una experiencia profesional con todas las exigencias de una iniciativa laboral real cuyos resultados se pueden contrastar con la realidad. En la práctica, el método se traduce en la realización de proyectos de investigación y planes de comunicación para entidades del tercer sector, llevados a cabo entre estudiantes de comunicación, profesores y profesionales de las entidades no lucrativas que participen en la iniciativa. Los tres actores forman un sistema colaborativo similar a la forma de trabajar de una agencia de comunicación con su cliente: los profesores son los supervisores de cuentas y las ENL adoptan el papel del cliente, mientras que los estudiantes forman el equipo profesional (ejecutivos de cuentas, creativos, directores de arte, investigadores, «community managers», etc.). Se trata de un sistema de aprendizaje proactivo, que responde a los intereses de los estudiantes, les educa en la faceta comunitaria de la comunicación (Abellán y Mayugo, 2008: 129-136) y anticipa las necesidades de comunicación de or- 
ganizaciones del tercer sector. Además es un método transversal, ya que permite la participación de alumnos procedentes de diferentes cursos y asignaturas.

\section{Desarrollo: descripción de los estudios sobre el método y aplicaciones}

Desde las primeras aplicaciones del «método docente de proyecto real» durante el curso 2003-4, el equipo lleva investigando la motivación y las opiniones de los alumnos participantes. Para una mayor validez de los resultados, se utilizó la triangulación metodológica, combinado el método cuantitativo con el cualitativo. En una primera fase exploratoria, se realizaron entrevistas informales para poder diseñar la fase conclusiva de la investigación: la encuesta sociológica, cuyos resultados se han completado y contrastado con la información aportada por los grupos de discusión. Debido al carácter longitudinal de la investigación, que se repite anualmente desde el curso 2004, se ha pretendido conocer si las opiniones y el nivel de conocimientos sobre métodos interactivos de los estudiantes han cambiado a lo largo de los años. Se ha utilizado el muestreo intencional debido a que al grupo de innovación le interesa conocer ante todo la visión de los estudiantes que recibieron docencia mediante un método interactivo, aunque también se incluyeron grupos de control ajenos al proyecto. Anualmente, se encuestaba a unos 200 estudiantes de comunicación y además, se llevaban a cabo unos 20 grupos de discusión. Debido a la gran cantidad de datos generados en la investigación, a continuación se resumirán únicamente los resultados globales de mayor interés para el presente tema, presentados en dos tramos temporales.

Entre las primeras muestras, de los cursos académicos 2004-2009, se percibió la interactividad de la siguiente forma:

- Para más del $80 \%$ de los alumnos encuestados, la interactividad se relacionó con la Web, especialmente con el Campus Virtual y en menor medida, con las TIC.

- Menos del 10\% la relacionó con la participación en clase y con la interacción con los docentes y el mundo profesional, mientras que el $10 \%$ restante no sabía definir el concepto (hasta 2007).

- A partir del curso 07/08, se empezó a relacionar también con la metodología relacionada con el nuevo EEES, pero sin que los alumnos supieran concretar en qué consistía.

\section{Significado de interactividad para los estudiantes (cursos 2004-2009)}

- Uso campus virtual y TICS

- Interacción social entre estudiantes, docentes y mundo profesional

- Aplicación Plan Bologna (desde 2007)

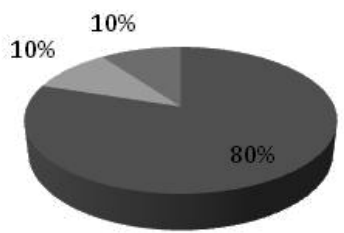

Gráfico 1: Muestra 2004-2009. El significado del método interactivo de docencia según los estudiantes. Fuente: elaboración propia. 
Las encuestas de los cursos 2010-2012 arrojaron resultados diferentes:

- Más del 90\% de los estudiantes encuestados asociaba la docencia interactiva con la interacción social entre profesores y estudiantes, y también, aunque en menor medida, con el mercado laboral.

- Fueron pocos los estudiantes (menos del 5\%) para quienes el método interactivo se relaciona únicamente con la Web, el campus virtual y las TIC, mientras que el $5 \%$ restante no contestaba.

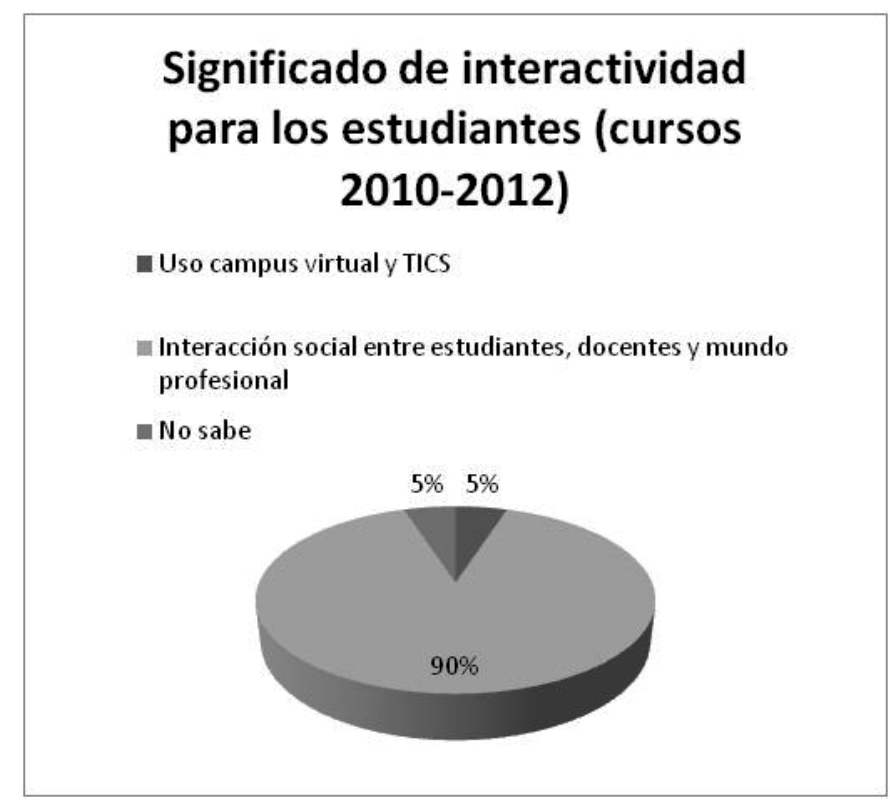

Gráfico 2: Muestra 2010-2012. El significado del método interactivo de docencia según los estudiantes. Fuente: Elaboración propia.

Los gráficos 1 y 2 señalan como la percepción de los estudiantes sobre el método interactivo ha variado durante los dos últimos cursos y como ahora se entiende como interacción social, mientras que hasta 2009 se asociaba con la interactividad tecnológica, y en caso concreto de los profesores, con el uso de las TIC y el campus virtual. Las técnicas cualitativas revelaron que este cambio se debe a la normalización del uso de la comunicación interactiva y digital en la docencia: se da por hecho la interacción tecnológica y por interacción se entiende lo social.

En las dos últimas encuestas, el $42 \%$ de los estudiantes ya habían recibido docencia mediante el método interactivo (interacción social) y sin embargo, sólo el 16\% opinaba que los profesores son interactivos. Para la gran mayoría (81\%) los profesores fueron poco interactivos desde el punto de vista social. En los grupos de discusión aparecieron como opiniones dominantes la falta del contacto social con los profesores y el escaso contacto con el mundo profesional. Los estudiantes se ven más preparados para manejar elementos virtuales que muchos profesores y reclaman un mejor 
aprovechamiento del Campus Virtual y de las herramientas digitales y relacionadas con el diseño. Cuando se les preguntó sobre el método de enseñanza ideal, hubo consenso respecto a las siguientes necesidades educativas:

- Combinación de la teoría con prácticas reales y profesionales.

- Más interés por los alumnos y mayor accesibilidad, más interacción con los estudiantes.

- Evaluación continuada y seguimiento constante, en vez de «jugar la nota de la asignatura» sólo con un examen.

- Aprovechamiento eficaz de las nuevas posibilidades de red y de las TIC, tanto desde el punto de vista de la enseñanza como herramientas estratégicas de comunicación.

\subsection{Aplicaciones del método las asignaturas}

La asignatura Técnicas de redacción de textos, que se imparte en la Facultad de Ciencias de la Información de la Universidad Complutense de Madrid, provee a los alumnos de los conocimientos teóricos básicos para analizar y comprender el mensaje publicitario, complementándose esta formación con permanentes ejercicios de redacción. Se seleccionó esta materia para la aplicación del método puesto que cuenta con un gran componente práctico, esencial a la hora de enseñar a los alumnos (estudiantes de segundo ciclo de Periodismo, Publicidad y Comunicación Audiovisual) el trabajo que realiza un redactor publicitario. Por ello, se quisieron aprovechar las ventajas que ofrecen los entornos virtuales para lograr implementar el modelo de enseñanzaaprendizaje propuesto en la metodología docente. En concreto, las herramientas empleadas han sido el blog y las redes sociales. La creación de la asignatura en Facebook y el blog han sido dos iniciativas fundamentales a la hora de favorecer la interactividad entre todos los componentes del proyecto, además de estar impulsadas plenamente por los alumnos.

El blog (http://copycreativos.blogspot.com), ha tenido como objetivos principales:

- Servir de plataforma a los alumnos de la asignatura, ayudándoles a que sus trabajos creativos puedan ser visualizados por profesionales de la publicidad, propiciando la incorporación de los estudiantes en el mundo laboral.

- Aplicar el método colaborativo a la dinámica del blog, mediante la valoración de las prácticas de los demás compañeros en los post individuales, realizando consideraciones y aportaciones constructivas que complementaran las clases teóricas.

- Incentivar el compromiso de los estudiantes con su propio aprendizaje, evaluando su proactividad en el blog.

- Sensibilizar a los universitarios en todos los aspectos relacionados con la importancia del trabajo en equipo y cooperativo.

Mediante la creación de la asignatura en Facebook se pretendía impulsar la doble interactividad del proyecto docente. Gracias a la red social, los interesados en conocer los trabajos de los alumnos, eran direccionados al blog donde se podían visualizar y escuchar. Así mismo, las agencias de publicidad y centros formativos y profesionales re- 
lacionados con la materia tenían la posibilidad de hacerse «amigos» y «fans» del trabajo de los alumnos, contribuyendo a su difusión. La función de relacionar a las empresas privadas e instituciones, con el blog y la página de facebook de nuestra asignatura, la está cumpliendo el docente. El problema de los derechos de autor de los estudiantes ante un posible plagio, se resolvió mediante una licencia propia que posee el blog y que protege la totalidad de los contenidos publicados en el mismo.

\subsection{Aplicaciones del método en la organización de un evento: III Convención África Existe}

La Convención África Existe (http://africa-existe.blogspot.com) es un evento íntegramente organizado por los estudiantes del segundo curso de Periodismo, que nace en el seno de la asignatura de Fundamentos de las Relaciones Públicas con un doble objetivo: 1) dar a conocer la realidad africana y sensibilizar a los estudiantes respecto a la complejidad del continente y 2) aprender de forma práctica las técnicas principales de las relaciones públicas. Es un ejemplo de «aprender haciendo», es una estrategia de comunicación en vivo y en directo, donde los alumnos trabajan durante cuatro meses para diseñar y llevar a cabo un evento real, de cuyo éxito dependerá su nota en la asignatura. Al principio del curso, se seleccionan los grupos de trabajo en función de los intereses y aptitudes personales de los estudiantes y así, se forman los equipos de dirección, investigación, creatividad, fotografia, vídeo, relaciones institucionales, prensa y redes sociales. La función de la profesora consiste en supervisar y corregir procedimientos, pero las decisiones se toman mediante consenso, para acostumbrar a los estudiantes a responsabilizarse de sus propias acciones. A continuación, los equipos comienzan a funcionar a modo de una agencia de comunicación, con sus sesiones de trabajo semanales, mientras que el resto de la clase investiga sobre diversas facetas de la realidad africana para luego dar a conocer los resultados de sus estudios mediante posters de convención e informes de prensa. La comunicación externa de la convención se organiza mediante «community management» en Facebook, Tuenti y Twitter, mientas que para la comunicación interna entre la organización se utilizaban más el campus virtual y la aplicación para móviles Whatsapp. La última edición de «África Existe» reunió a más de 500 alumnos de la facultad y contó con la presencia de una docena de entidades no lucrativas, entre ellos ACNUR y Reporteros sin Fronteras. Los alumnos lograron que participasen con ponencias periodistas conocidos, entre otros la reportera Erika Reija de TVE y el veterano fotoperiodista Enrique Meneses, además de conseguir que una treintena de medios de comunicación hicieran eco del evento.

\section{Conclusiones}

Como resultado de la labor docente e investigadora efectuada, así como del análisis de las repercusiones sociales y pedagógicas de los proyectos reales que el grupo de innovación ha desarrollado, concluimos que:

1. La aplicación de conocimientos y habilidades mediante proyectos sociales y culturales reales cada curso, ha supuesto que más de 500 alumnos hayan aplicado sus conocimientos en iniciativas reales de comunicación. 
2. Contactar con el tercer sector ayudó a que los estudiantes hayan trabajado para más de 50 ENLS, propiciando así su incorporación al mercado laboral.

3. Se han establecido redes de colaboración entre alumnos, profesores y profesionales. Los alumnos asumieron el papel de ejecutivos de comunicación, los profesores el de supervisores de cuentas y las entidades colaboradoras el de clientes (agencias de comunicación).

4. Se ha fomentado el espíritu altruista de los alumnos, educándoles en valores solidarios: para todos los alumnos participantes, el hecho de trabajar con entidades no lucrativas y profundizar en temas sociales graves, tales como la pobreza, la marginación, las enfermedades y la discriminación, les ha permitido reflexionar sobre la realidad social. Se ha generado cierto "efecto de bola de nieve", ya que los alumnos participantes han compartido experiencias con otros estudiantes y durante el presente curso, ha aumentado la demanda de participación en las diversas iniciativas.

5. Se ha obtenido una retroalimentación informativa inmediata o a medio plazo en la sociedad local y las regiones menos favorecidas de los países en vías de desarrollo: el feedback de las entidades participantes respecto a los resultados de las campañas elaboradas por los estudiantes ha sido mayoritariamente positiva hasta el momento. Sin embargo, en función de las necesidades de las entidades colaboradoras, se corrigen los aspectos mejorables de las iniciativas.

6. El proyecto ha garantizado una experiencia profesional a los alumnos, pero además, estudiantes que han participado en años anteriores han comentado que su colaboración con las ENLS se ha valorado de forma muy positiva en los procesos de selección posteriores (testimonios de profesionales de Procter \& Gamble, Publicis, Google).

7. El método de proyecto social real ha supuesto un cambio de mentalidad respecto a los sistemas tradicionales y unidireccionales de enseñanza y se adapta perfectamente a los requisitos del EEES.

8. Se ha aumentado la responsabilidad social corporativa de la universidad: por si sólo, el presente proyecto ha generado una treintena de convenios de colaboración con ENLS para la Universidad Complutense, llenos de contenido y que se han convertido en iniciativas reales con resultados contrastables. A raíz de este tipo de iniciativas, la Facultad de Ciencias de la Información de la UCM empieza a posicionarse y puede convertirse en un referente de responsabilidad corporativa, al tratarse de un proyecto cada vez más conocido en el área de las ONGS y ENLS.

\section{Referencias bibliográficas}

ABELLÁN, Gemma y MAYUGO, Carmen (2008): "La dimensión comunitaria de la educación en comunicación". Comunicar, $\mathrm{n}^{\circ} 31$. Madrid, Editorial Grupo Comunicar, pp. 129-136.

CASTELLS, Manuel (2009): Communication power. Nueva York, Oxford University Press. 
CONSEJO DE COORDINACIÓN UNIVERSITARIA (2006): "Propuesta para la renovación de las metodologías educativas en la universidad". Informe Fundación Coordinación Universidades. Madrid, Secretaría General Técnica del Ministerio de Educación y Ciencia.

GARCÍA ARETIO, Lorenzo (2001): La educación a distancia. De la teoría a la práctica. Barcelona, Ariel Educación.

JOHNSON, David y JOHNSON, Roger (1999): Learning together and alone. Boston, Allan \& Bacon.

KOLB, David .A. (1984): Experiential Learning: experience as the source of learning and development. New Jersey, Prentice-Hall.

NÚÑEZ, Patricia y HÄNNINEN, Liisa (2010): “The methodology of real social projects applied in NGOS: Latest advances carried out at the Faculty of Communication of the Complutense University of Madrid". Edulearn10 Proceedings, Barcelona, IATED, pp. 4416-4426.

SAURA, Pilar et al. (2007): La experiencia solidaria y educativa de las Operaciones Kilo. Madrid, Publicis Healthcare Commmunications Group.

UNESCO (1995): Documento de política para el cambio y desarrollo de la educación Superior. París, UNESCO.

UNESCO (1998): La educación superior en el siglo XXI. Visión y acción. París, UNESCO.

\section{Tamara BUENO DORAL}

trbueno@pdi.ucm.es

Facultad de Ciencias de la Información de la UCM, despacho 419

Avda. Complutense SN, Ciudad Universitaria, 28040 Madrid

Profesora ayudante

Facultad de Ciencias de la Información de la Universidad Complutense de Madrid.

Doctora en Ciencias de la Información, especialista en género y comunicación para el desarrollo.

\section{Liisa Irene HÄNNINEN}

liisaire@pdi.ucm.es

Facultad de Ciencias de la Información de la UCM, despacho 01 bis

Avda. Complutense SN, Ciudad Universitaria, 28040 Madrid

Profesora contratada doctora

Facultad de Ciencias de la Información de la Universidad Complutense de Madrid.

Doctora en Ciencias de la Información, especialista en comunicación social, relaciones pú-

blicas e investigación para el tercer sector. Universidad Complutense de Madrid 